\title{
Adsorption potentials for nonplanar geometries
}

\author{
A. Hernando, ${ }^{1}$ E. S. Hernández, ${ }^{2}$ R. Mayol, ${ }^{1}$ and $\mathrm{M} . \mathrm{Pi}^{1}$ \\ ${ }^{1}$ Departament d'Estructura i Components de la Matèria, Facultat de Física, and IN ${ }^{2}$ UB, \\ Universitat de Barcelona, 08028 Barcelona, Spain \\ ${ }^{2}$ Departamento de Física, Facultad de Ciencias Exactas y Naturales, Universidad de Buenos Aires, \\ Ciudad Universitaria, Pabellón I, 1428 Capital Federal, Buenos Aires, Argentina \\ and Consejo Nacional de Investigaciones Científicas y Técnicas, Avenida \\ Rivadavia 1917, Codigo Postal C1033AAJ, Buenos Aires, Argentina
}

(Received 2 May 2007; revised manuscript received 12 July 2007; published 21 September 2007)

\begin{abstract}
We present a method to compute, assuming a continuous distribution of sources, the elementary potential created by a differential element of volume of matter, whose integral generates a known adsorption field $V(z)$ for a planar surface. We show that this elementary potential is univocally determined by the original field and can be used to generate adsorption potentials for other nontrivial geometries. We illustrate the method for the Chizmeshya-Cole-Zaremba physisorption potential and discuss several examples and applications.
\end{abstract}

DOI: $10.1103 /$ PhysRevB.76.115429

PACS number(s): 68.08.Bc, 68.08.- p, 68.65.-k, 68.35.Np

\section{INTRODUCTION}

One important field of research in low temperature physics is adsorption of quantum fluids on surfaces of various geometries. For most nontrivial shapes of the substrate, the lack of a reliable and easy-to-use adsorption potential represents a serious limitation for calculational purposes. Traditionally, this difficulty has been sorted out in essentially two ways. One is to compute the integral of a three-dimensional (3D) adatom-adsorber pairwise interaction over the specified geometry, as carried out for planar, ${ }^{1-6}$ cylindrical, ${ }^{7}$ and spherical ${ }^{8}$ substrates. The other approach, oriented to geometries presenting angles, i.e., wedges and polygonal pores, is to compose the adsorbing field by summing up the contributions from the participating walls. ${ }^{9-13}$ While the former procedure is of limited accuracy in the case of highly structured substrates such as graphite, the second can be shown to overestimate the adsorption strength near vertices due to double counting from the region where the half solids overlap. Notwithstanding these limitations, the physics of confined quantum fluids has largely benefited from the various approximations to trace general trends and anticipate on several interesting phenomena, such as prewetting transitions on planar substrates and pore and wedge filling. However, the choice of the force parameters such as well depth and interaction range is critical when determining characteristics of film growth, atom dynamics, and phase diagram of the adsorbed fluid. ${ }^{12}$ It is then useful to develop a tool to derive the most accurate, trustworthy potential for the given confining, nonplanar environment.

The Chizmeshya-Cole-Zaremba (CCZ) physisorption potential ${ }^{14}$ for noble gases and hydrogen on metal surfaces is, up to now, the best suited for studies of wetting of planar substrates. These authors have provided the field $V_{C C Z}(z)$ created by a semi-infinite solid material at distance $z$ from the free surface. The construction of this family of potentials is carried out by adding dispersion and overlap contributions, both determined $a b$ initio. The result is then fitted to a simple functional form, namely,

$$
V_{C C Z}(z)=V_{0}(1+\alpha z) e^{-\alpha z}-f_{2}\left[\beta(z)\left(z-z_{v d W}\right)\right] \frac{C_{v d W}}{\left(z-z_{v d W}\right)^{3}},
$$

with $f_{2}(x)=1-e^{-x}\left(1+x+x^{2} / 2\right)$ and $\beta(z)=\alpha^{2} z /(1+\alpha z)$, which is used in this paper. In view of its physical origin and analytical form, this potential cannot, in principle, be decomposed as a summation over pair interactions between adsorbate and atoms in the adsorber. However, we may intuitively think on purely geometrical grounds that, if all the differential volume elements in the material contribute to the adsorption potential at $z$ with a weight depending only on the distance to the adatom, the total strength $V_{C C Z}(z)$ can be obtained by an integration over a continuous half solid. In such a case, one can design a method to obtain that elementary potential from the adsorption field.

In this work, we show that given a physisorption potential for a planar surface of the form $V(z)$, under very general assumptions, it is possible to decompose it into contributions from elementary geometric sources located in the bulk of the material. Once these generators are identified, appropriate recombination into an integral for an arbitrary geometry permits us to simulate a distortion of the original planar surface and obtain the corresponding two-or three-dimensional adsorption potential. The general method is described in Sec. II. In Sec. III, we present several illustrations of the general procedure and discuss aspects of the condensation of helium in various geometries. The conclusions and perspectives are summarized in Sec. IV.

\section{METHOD}

For arbitrary geometries, the potential $V$ created by a continuous distribution of matter $\rho\left(\mathbf{r}^{\prime}\right)$ on an atom placed at $\mathbf{r}=(x, y, z)$ is given by the convolution

$$
V(\mathbf{r})=\int d \mathbf{r}^{\prime} \rho\left(\mathbf{r}^{\prime}\right) v\left(\mathbf{r}^{\prime}-\mathbf{r}\right)
$$


where the generating kernel $v(\mathbf{x})$ is defined here as the $\mathrm{el}$ ementary potential. For a continuous half solid with free surface at position $z^{\prime}=0$, we have $\rho\left(\mathbf{r}^{\prime}\right)=\rho_{0} \Theta\left(-z^{\prime}\right)$, where $\rho_{0}$ is the bulk density of the material and $\Theta(x)$ the usual Heavyside function. Hereafter, we adopt a reference potential $V_{\text {ref }}(z)$, for example, the $\mathrm{CCZ}$ one, and assuming that $v$ depends only on the distance, the integral is

$$
V_{\text {ref }}(z)=\rho_{0} \int_{-\infty}^{\infty} d x^{\prime} \int_{-\infty}^{\infty} d y^{\prime} \int_{z}^{\infty} d \zeta v(R),
$$

with $\zeta=z-z^{\prime}$ and $R=\sqrt{x^{\prime 2}+y^{\prime 2}+\zeta^{2}}$. In this expression, we have explicitly taken advantage of the translational invariance of the system on the horizontal plane.

Choosing polar coordinates $\left(r^{\prime}, \varphi^{\prime}\right)$ in the $\left(x^{\prime}, y^{\prime}\right)$ plane, Eq. (3) becomes

$$
V_{r e f}(z)=2 \pi \rho_{0} \int_{z}^{\infty} d \zeta \int_{\zeta}^{\infty} d R R v(R) .
$$

The latter line indicates that in this case, the elementary sources for the potential at $z$ are parallel planes in the bulk of the adsorber, at vertical distance $\zeta$ from the test particle. Other selection of coordinates allows one to identify sources of various shapes such as parallel filaments and cylindrical or spherical surfaces. Moreover, since Eq. (4) is an integral equation to be solved under adequate boundary conditions, it can be proven that if $v(R)$ is requested to vanish at infinity, it is unique.

For this sake, we note that double differentiation of Eq. (3) with respect to $z$ gives

$$
V_{r e f}^{\prime \prime}(z)=2 \pi \rho_{0} z v(z) .
$$

In fact, this is a representation of Poisson's equation for the distribution of elementary sources: Computing the Laplacian of the convolution [Eq. (2)] and switching integration variables, one has

$$
\nabla^{2} V(\mathbf{r})=\int d \mathbf{r}^{\prime} \nabla^{2} \rho\left(\mathbf{r}-\mathbf{r}^{\prime}\right) v\left(\mathbf{r}^{\prime}\right) .
$$

Introducing the matter density of a semi-infinite solid, the above equation becomes

$$
\frac{d^{2} V_{r e f}(z)}{d z^{2}}=\int d \mathbf{r}^{\prime} \delta^{\prime}\left(z-z^{\prime}\right) v\left(\mathbf{r}^{\prime}\right) .
$$

Integration by parts with appropriate choice of variables immediately leads to Eq. (5), which clearly shows that for a given $V_{\text {ref }}(z)$, the elementary potential is univocally and simply defined; it naturally satisfies the necessary boundary condition of vanishing at large distances [cf. Eq. (1)] and can be applied to arbitrary geometries, writing the distance $R$ between adatom and adsorber in an adequate coordinate system and multiplying by the proper weighting function. Computationally, Eq. (5) and the subsequent integration given by Eq. (4) are very fast and precise.

For matter with constant density $\rho_{0}$ and an arbitrary surface defined by a function $z=f(x, y)$, Eqs. (2) and (5) give the potential $V(\mathbf{r})$ on a test particle as
TABLE I. Minimum of the elementary CCZ potential and its position for a $\mathrm{He}$ atom and $\mathrm{a}_{2}$ molecule.

\begin{tabular}{lccccc}
\hline \hline & \multicolumn{2}{c}{$\mathrm{He}$} & & \multicolumn{2}{c}{$\mathrm{H}_{2}$} \\
\cline { 2 - 3 } \cline { 5 - 6 } Species & $\begin{array}{c}\rho_{0} v_{C C Z}\left(r_{\text {min }}\right) \\
\left(\mathrm{K}^{-3}\right)\end{array}$ & $\begin{array}{c}r_{\text {min }} \\
(\AA)\end{array}$ & & $\begin{array}{c}\rho_{0} v_{C C Z}\left(r_{\text {min }}\right) \\
\left(\mathrm{K}^{-3}\right)\end{array}$ & $\begin{array}{c}r_{\text {min }} \\
(\AA)\end{array}$ \\
\hline $\mathrm{Mg}$ & -0.214 & 4.72 & & -1.556 & 4.03 \\
$\mathrm{Li}$ & $-7.22 \times 10^{-2}$ & 5.38 & & -0.544 & 4.60 \\
$\mathrm{Na}$ & $-4.31 \times 10^{-2}$ & 5.65 & & -0.324 & 4.82 \\
$\mathrm{~K}$ & $-2.04 \times 10^{-2}$ & 6.17 & & -0.153 & 5.25 \\
$\mathrm{Rb}$ & $-1.67 \times 10^{-2}$ & 6.33 & & -0.125 & 5.37 \\
$\mathrm{Cs}$ & $-1.55 \times 10^{-2}$ & 6.34 & -0.117 & 4.97 \\
\hline \hline
\end{tabular}

$$
V(\mathbf{r})=\int \frac{d \mathbf{r}^{\prime}}{2 \pi} \Theta\left[f\left(x^{\prime}, y^{\prime}\right)-z^{\prime}\right] \frac{V_{r e f}^{\prime \prime}\left(\left|\mathbf{r}-\mathbf{r}^{\prime}\right|\right)}{\left|\mathbf{r}-\mathbf{r}^{\prime}\right|} .
$$

In particular, the restriction to a half solid,

$$
V_{r e f}(z)=\int \frac{d \mathbf{r}^{\prime}}{2 \pi} \Theta\left[-z^{\prime}\right] \frac{V_{r e f}^{\prime \prime}\left(\left|\mathbf{r}-\mathbf{r}^{\prime}\right|\right)}{\left|\mathbf{r}-\mathbf{r}^{\prime}\right|},
$$

permits us to define the "planar potential" $V(z)$ as a solution of this integral equation. It is straightforward to prove that the widely used 3-9 potentials derived from the LennardJones (LJ) pair interaction ${ }^{1-6,10-12}$ belong to this class.

As a first test, we have computed the elementary potentials $v_{C C Z}(r)$ corresponding to the $\mathrm{CCZ}$ adsorption fields for $\mathrm{He}$ atoms and $\mathrm{H}_{2}$ molecules on various metallic substrates. The minimum values $v_{C C Z}\left(r_{\min }\right)$ and their positions are listed in Table I, and the elementary potentials are plotted in the upper panel of Fig. 1. The lower panel shows the CCZ potentials (full lines) together with the elementary potentials integrated according to Eq. (4) using a Gauss quadrature (symbols). From this figure, it can be seen that the original CCZ potential is recovered after integration over continuous differential elements of matter.

The application to the CCZ potential serves as well the purpose of illustrating the limitation of the method. In fact, $v_{C C Z}(R)$ is negative for $\alpha R<1$ [cf. Eq. (1)]. This is not visible in the scale of Fig. 1(a) since for all metals in Table I, the short-range parameter $\alpha$ lies between 1.77 and $2.66 \AA$ (Ref. 1); the striking convergence among points and lines in Fig. 1(b) indicates that this apparent drawback is irrelevant upon integration over large samples of matter. Similarly, the long-range scale $z_{v d W}$ of the CCZ potentials-between 0.19 and $0.34 \AA$-introduces another limitation. Given these two natural lengths of the CCZ potentials, and their typical values, it appears that the present method can be safely employed insofar as integrations are carried over samples of matter not smaller than around $\alpha_{\min }^{-1}=0.56 \AA$. Similar care should be taken for any reference potential under use.

\section{SOME RESULTS FOR He ADATOMS}

We have performed integrations of the elementary CCZ potentials for helium atoms for a variety of shapes. In current 

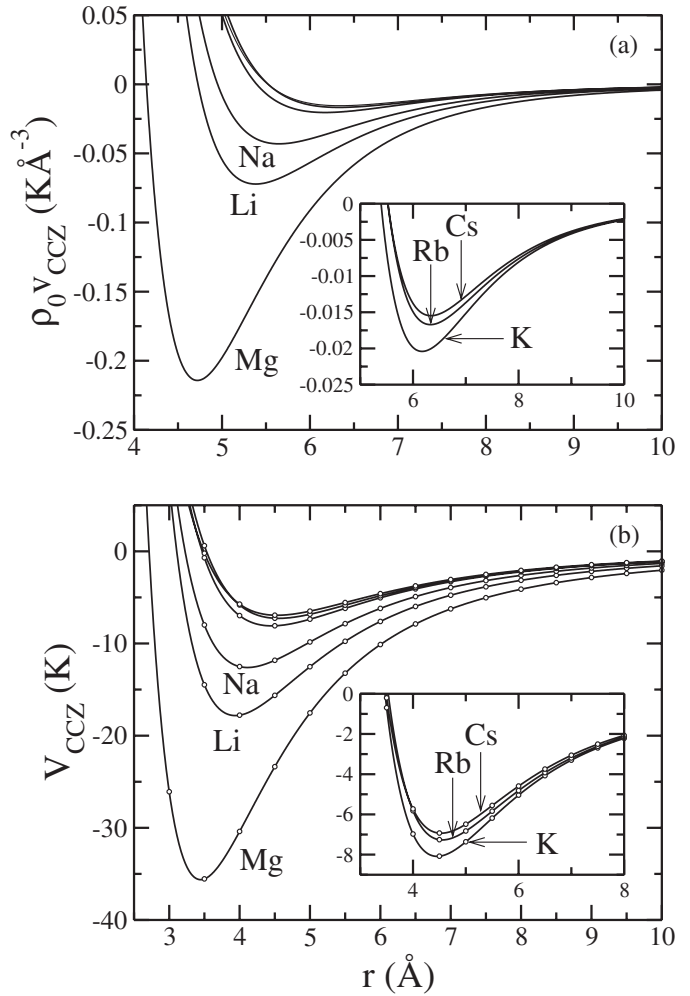

FIG. 1. (a) Elementary potential $\rho_{0} v_{C C Z}(r)$ for a He atom in the presence of several metal substrates; (b) the CCZ potentials (full lines) and the integrals of the elementary potentials from the upper plot (symbols). The insets span the neighborhood of zero potential.

research of wetting by quantum fluids, interesting geometries are hollow cylinders of infinite length with different transverse sections that simulate pores inside bulk material. For circular cylinders, the potentials have been computed from LJ pair interactions, ${ }^{7}$ and for geometries including angles, such as the hexagonal pore in Refs. 10 and 12, the confining field has been represented as the summation of six 3-9 potentials. In addition, a cusp of solid material may provide a tool to model some irregularities on rough surfaces. As an illustration, in the lower and upper panels of Fig. 2, we, respectively, display the $(x, z)$ contour plot of the integrated potential for a Cs solid cusp and for an hexagonal pore in bulk Cs, in both cases with translational invariance along the $y$ axis. It is interesting to note that the hexagonal pore field can be computed summing six nonoverlapping solid cusps, with walls indicated by the dotted lines in the plot. The potential landscapes clearly reveal the expected condensation patterns that, for small amounts of helium, consist of one quasi-two-dimensional layer on either cusp wall and of one quasi-one-dimensional stripe at each vertex of the hexagon. The latter is consistent with the condensation pattern encountered in Ref. 12.

In Fig. 3, we show the potentials inside infinite cylindrical cavities of radius $R=10 \AA$ in bulk $\mathrm{Cs}, \mathrm{Li}$, and $\mathrm{Mg}$, plotted as a function of radial distance, together with those computed as in Ref. 16 out of integration of a LJ potential. The parameters $\varepsilon$ and $\sigma$ from Ref. 8 have been fixed so as to reproduce the depth of the corresponding planar CCZ field. We note that although quite similar in shape and scales, the CCZ-
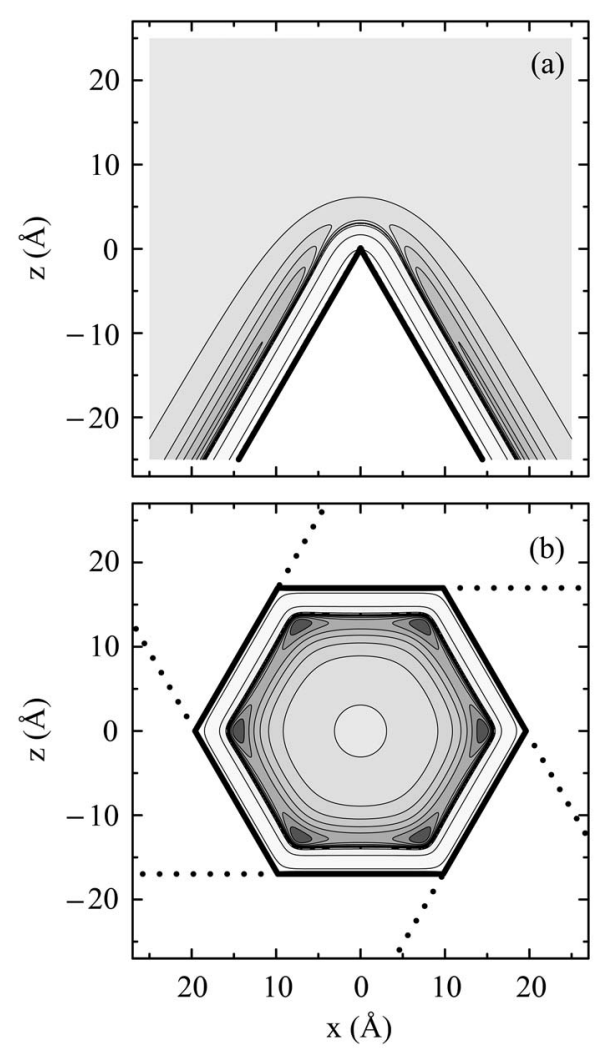

FIG. 2. (a) Potential landscape for an infinite Cs cusp; (b) same for an infinite hexagonal pore in bulk Cs. The solid walls are indicated with dark lines.

based potentials for the strong adsorbers $\mathrm{Li}$ and $\mathrm{Mg}$ are moderately deeper and the minima are slightly shifted out of the tube wall, as compared with the LJ ones.

The method here proposed can demonstrate that for pores with polygonal section, the summation of planar potentials may be a reliable approximation far from a vertex, whereas they overestimate the adsorption strength substantially in the vicinity of an apex. As an illustration, we consider a rhombic pore with angles of $60^{\circ}$ and $120^{\circ}$, extending infinitely along the $y$ axis in bulk Cs. Since such a pore can be viewed as a

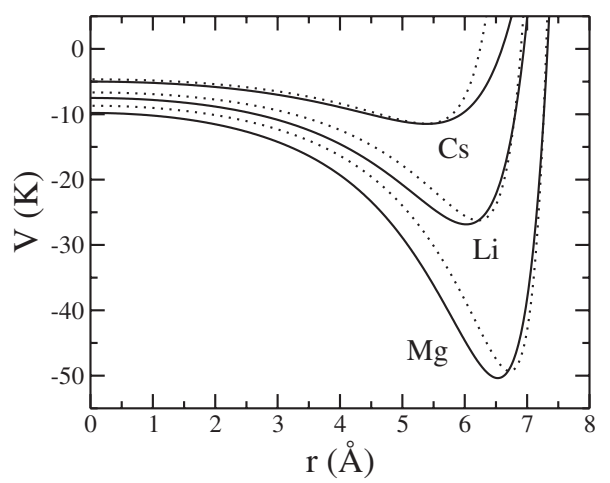

FIG. 3. Integrated $\mathrm{CCZ}$ potentials for a $\mathrm{He}$ atom inside infinite cylindrical pores of radius $R=10 \AA$ in bulk $\mathrm{Cs}, \mathrm{Li}$, and $\mathrm{Mg}$, compared with the ones derived from a LJ pair interaction (full and dotted lines, respectively). 

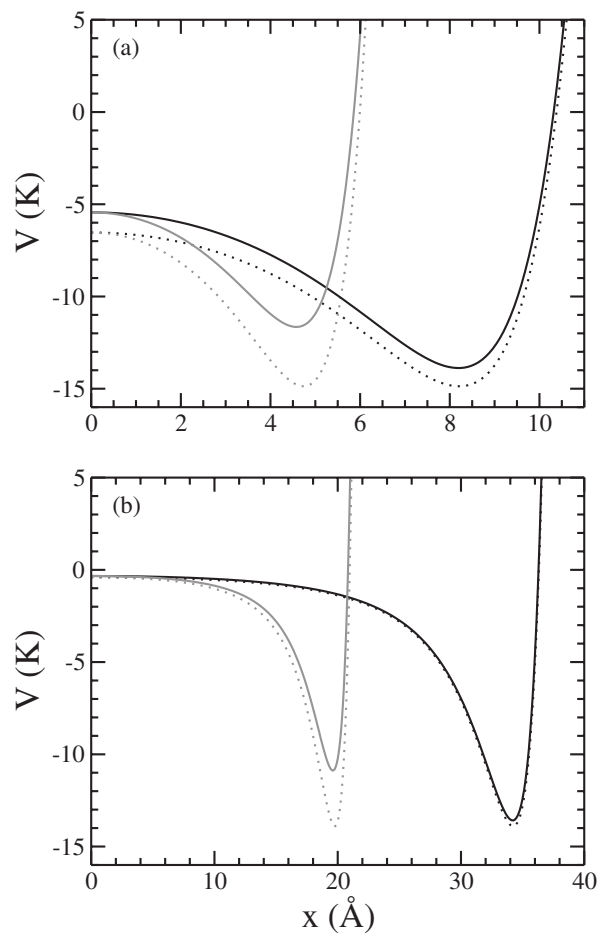

FIG. 4. Integrated elementary potential $V_{\text {rhombus }}(x, z)$ along the diagonals of a hollow rhombus of sides (a) $20 \AA$ and (b) $50 \AA$, both with angles of $60^{\circ}$ and $120^{\circ}$ in bulk Cs (full lines), compared with the adsorption potential generated by four planar $\mathrm{CCZ}$ fields at the indicated angles (dotted lines). The coordinate origin is at the center of the rhombus, and $x$ is the distance along each diagonal. Dark (gray) curves correspond to the longest (shortest) diagonal.

symmetric array of two wedges of finite height, forming four inner apertures, it is clear that for small amounts of helium, one should expect condensation near the vertices. In order to visualize the enhancement of the physisorption strength, in Fig. 4, we represent the integrated elementary potential, together with the summation of four planar CCZ fields, along the diagonals of pores with sides of 20 and $50 \AA$, respectively, in the upper and lower panels. We realize that the overestimation effects are sizable at the weakest minima corresponding to the vertices joined by the shortest diagonal, where the depth is increased by $5-6 \mathrm{~K}$, as opposed to less than $2 \mathrm{~K}$ in the large diagonal of the small pore. The potentials are essentially indistinguishable along the longest diagonal of the large pore.

Another interesting illustration is a 3D conical pore. Figure 5 shows the equipotential lines of such structure on the $(r, z)$ plane for apertures of $120^{\circ}$ and $60^{\circ}$ and a depth of $50 \AA$. This potential landscape on a symmetry plane is qualitatively similar to that corresponding to a linear wedge with finite height, and far enough from the pore, we recover the CCZ potential of the planar surface. This example combines a wedgelike and a cusplike behavior, as seen in the contours, and indicates that within vertical heights of the order of the position of the minimum, where condensation of the adsorbate takes place for not too large amounts of helium, boundary effects can be safely neglected.

Finally, we revise the problem of condensation of helium in wedges. ${ }^{13}$ In Fig. 6, we illustrate the density profile along
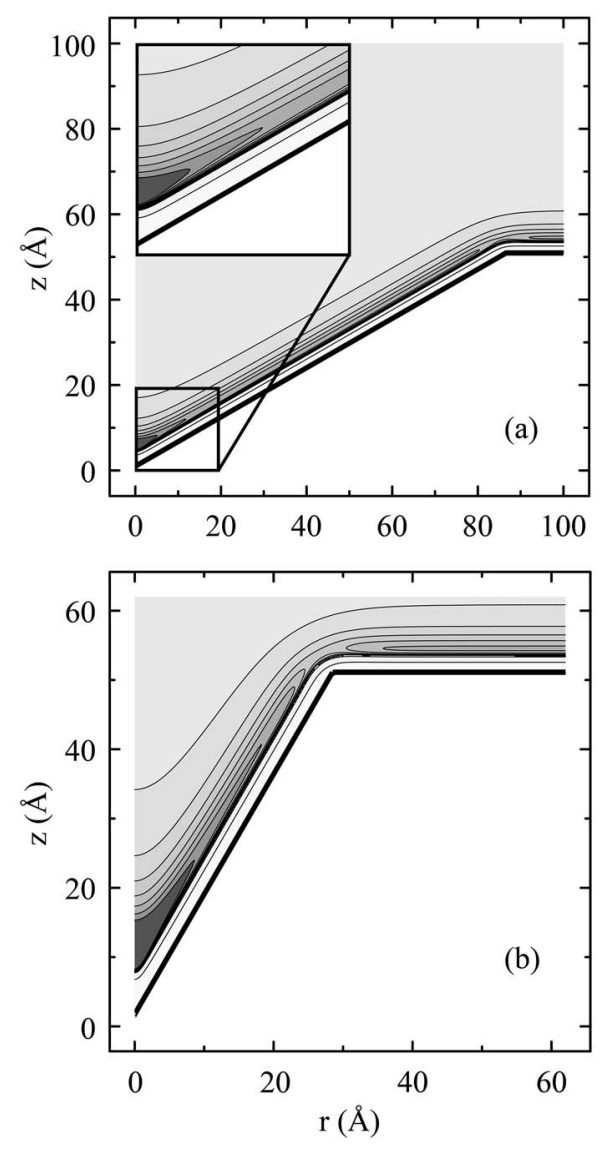

FIG. 5. Integrated potential $V_{\text {cone }}(r, z)$ for a conical hole with $50 \AA$ depth on a symmetry plane. (a) Aperture of $120^{\circ}$; (b) aperture of $60^{\circ}$.

the $z$ axis (upper panel) and the equidensity lines corresponding to half the saturation density of ${ }^{4} \mathrm{He}$ (lower panel) in an infinite wedge with aperture of $170^{\circ}$, for a linear density of $140 \AA^{-1}$. Full and dotted lines correspond to the wedge potential generated by the elementary CCZ sources and to the summation of two planar CCZ fields as done in Ref. 13. We see in the upper figure that double counting at the apex enhances the height of the main peak by nearly $25 \%$ and compresses the sample on the vertical axis by $2-3 \AA$. This enhancement is totally attributed to the extra attraction near the vertex provoked by the overlapping planes. Both representations of the potential agree in the location of the density peaks and coincide at the minima of the density oscillations. The shape of the sample in the lower plot also shows an overall compression of the meniscus, compensated by some spreading of the helium fluid near the contact line. Note that for this large opening, double-counting effects in the density profiles, however moderate, are visible; a similar comparison carried at, i.e., $45^{\circ}$ gives almost indistinguishable fluid distributions. This behavior is supported by a glance at the energetic features displayed in Table II, where we show the energy per particle, chemical potential, and grand potential per particle, $\Omega / N=E / N-\mu$, for helium atoms on planar Cs and on Cs wedges of $170^{\circ}$ and $45^{\circ}$, the latter being computed under the summation approximation and by integration of the CCZ elementary potentials. 

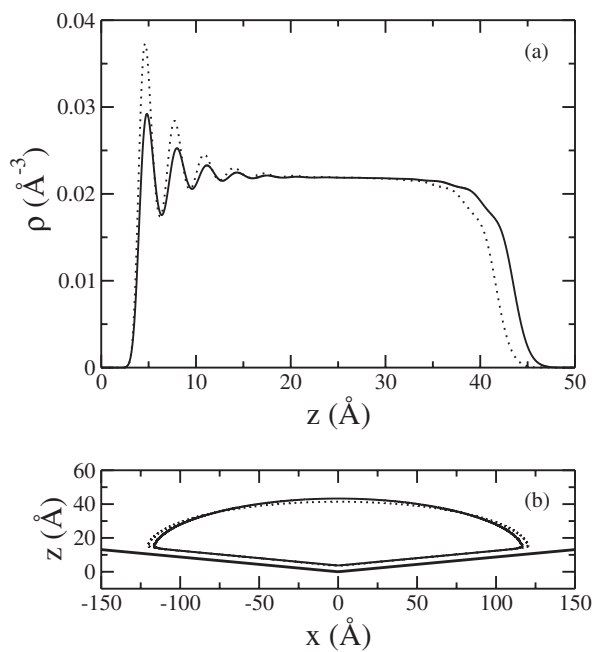

FIG. 6. (a) Density profile on the $z$ axis of a wedge with aperture of $170^{\circ}$; (b) equidensity contour at half the saturation density of bulk ${ }^{4} \mathrm{He}$. Full and dotted lines, respectively, correspond to the integrated CCZ elementary potential and to the summation of two planar CCZ potentials. The linear atom density is $140 \AA^{-1}$.

These results show that folding a plane into an angle of $170^{\circ}$ is, in fact, a minor perturbation provided that the adsorption field is properly computed, since all relevant energetic features remain essentially unchanged. By contrast, the summation of two planar fields is a poor approximation that changes the wetting behavior from nonwetting $(\Omega / N>0)$ to wetting $(\Omega / N<0)$. Instead, for a not-too-small angle such as $45^{\circ}$, both representations of the adsorption field yield equivalent results. This identity between results provided by the two different representation of the wedge potential persists for even smaller angles at which bridge configurations of the helium atoms appear. ${ }^{13}$ It is also worth noting that in the present calculation, for either potential, all wedge configurations are thermodynamically stable with respect to a droplet with same linear density lying on one planar wall (aperture of $180^{\circ}$ ), since the latter carries the highest (nonwetting) grand potential per particle. These properties are important in view of the different adsorption isotherms at fixed angle ${ }^{15}$ that can be expected under these two methods; an inadequate choice of the potential might shift the condensation threshold

TABLE II. Energy per particle, chemical potential, and grand potential per particle for helium atoms on planar Cs and on Cs wedges of $170^{\circ}$ and $145^{\circ}$, computed by integration of the $\mathrm{CCZ}$ elementary potentials and by summation of two planar CCZ potentials.

\begin{tabular}{lccc}
$\begin{array}{l}\text { Aperture } \\
\text { (deg) }\end{array}$ & $E / N(K)$ & $\mu(K)$ & $\Omega / N$ \\
\hline 180 & -7.07 & -7.13 & 0.06 \\
170 (integrated CCZ) & -7.08 & -7.13 & 0.05 \\
170 (two CCZ planes) & -7.29 & -7.16 & -0.13 \\
45 (integrated CCZ) & -7.42 & -7.31 & -0.12 \\
45 (two CCZ planes) & -7.42 & -7.31 & -0.12 \\
\hline \hline
\end{tabular}

to lower linear densities in the wedge. This is the case for hexagonal silica and metallic pores. ${ }^{18}$ In particular, the approximation under which the calculations in Ref. 13 were performed is perfectly reliable, since only the aperture range below $120^{\circ}$, where the interesting phenomena concerning filling and emptying transitions appear to take place, was considered there.

\section{SUMMARY}

In this work, we have proposed a means to derive the elementary potentials that create a given planar adsorption potential, assuming a continuous distribution of the sources. The output can afterward be integrated weighted with a density that corresponds to an arbitrary geometry to obtain the adsorption potential for a semi-infinite solid with a nonplanar solid-fluid interface. The method is numerically fast and robust and gives rise to potential landscapes that account adequately for the irregularities of the surface, as viewed in several illustrations presented in this work. For specific geometries with particular invariances, the integration involves one or two dimensions and eventually reduces, either partially or totally, to some analytical expression.

All applications presented in this paper invoke one class of planar potentials, the CCZ ones, derived for metallic substrates. These are $a b$ initio potentials that cannot, in principle, be represented by summation or integration of a pair interaction between adatom and adsorbate atom over the substrate. However, in many situations of experimental and theoretical interest, the material exposed to the helium vapor is a glass or a gel of silica strands. ${ }^{6,11,12}$ In these cases, the only planar adsorption potential available is the 3-9 one, and the adopted procedure to derive the field inside a polygonal pore is summation of planes. ${ }^{10,12}$ Integration of the elementary potential, which for the 3-9 field amounts to integrating the original LJ pair interaction, as shown in this paper, could substantially improve the description avoiding undesired double-counting effects in the vicinity of vertices. ${ }^{18}$

Some of the geometries presented here as illustrations can be viewed as "folding" or deformations of a planar interface. With the construction of a cusp and a conical pore, we show that with this procedure, one can mimic irregularities in a planar substrate, where approximations based on summation of planar potentials cannot be applied. This is especially promising with the perspective of modeling rough or patterned surfaces ${ }^{17}$ keeping the most important physical characteristics of the planar one that gives rise to the elementary potential.

\section{ACKNOWLEDGMENTS}

We are indebted to Francesco Ancilotto, Manuel Barranco, and Milton Cole for helpful discussions and useful comments on this paper. This paper was performed under Grants No. FIS2005-01414 MEC-DGI and No. 2005SGR00343 Generalitat de Catalunya (Spain), PIP 5138/05 from CONICET, and X298 from University of Buenos Aires (Argentina). 
${ }^{1}$ E. Cheng, M. W. Cole, W. F. Saam, and J. Treiner, Phys. Rev. Lett. 67, 1007 (1991).

${ }^{2}$ N. Pavloff and J. Treiner, J. Low Temp. Phys. 83, 331 (1991).

${ }^{3}$ E. Cheng, M. W. Cole, W. F. Saam, and J. Treiner, Phys. Rev. B 48, 18214 (1993).

${ }^{4}$ E. Cheng, M. W. Cole, J. Dupont-Roc, W. F. Saam, and J. Treiner, Rev. Mod. Phys. 65, 557 (1993).

${ }^{5}$ J. Treiner, J. Low Temp. Phys. 92, 1 (1993).

${ }^{6}$ V. Apaja and E. Krotscheck, Phys. Rev. B 67, 184304 (2003); Phys. Rev. Lett. 91, 225302 (2003).

${ }^{7}$ G. Stan and M. W. Cole, Surf. Sci. 395, 280 (1998).

${ }^{8}$ E. S. Hernández, M. W. Cole, and M. Boninsegni, Phys. Rev. B 68, 125418 (2003).

${ }^{9}$ E. Cheng and M. W. Cole, Phys. Rev. B 41, 9650 (1990).

${ }^{10}$ M. W. Cole, F. Ancilotto, and S. M. Gatica, J. Low Temp. Phys. 138, 195 (2005).
${ }^{11}$ M. Rossi, D. E. Galli, and L. Reatto, J. Low Temp. Phys. 146, 95 (2006).

${ }^{12}$ M. Rossi, D. E. Galli, and L. Reatto, Phys. Rev. B 72, 064516 (2005).

${ }^{13}$ E. S. Hernández, F. Ancilotto, M. Barranco, R. Mayol, and M. Pi, Phys. Rev. B 73, 245406 (2006).

${ }^{14}$ A. Chizmeshya, M. W. Cole, and E. Zaremba, J. Low Temp. Phys. 110, 677 (1998).

${ }^{15}$ R. Mayol, F. Ancilotto, M. Barranco, E. S. Hernández, and M. Pi, J. Low Temp. Phys. 148, 851 (2007).

${ }^{16}$ E. S. Hernández, J. Low Temp. Phys. 137, 89 (2004).

${ }^{17}$ J. Klier, P. Leiderer, D. Reinelt, and A. F. G. Wyatt, Phys. Rev. B 72, 245410 (2005).

${ }^{18}$ E. S. Hernández, A. Hernando, R. Mayol, and M. Pi, in Proceedings of the 14th International Conference on Recent Progress in Many-Body Theory, Barcelona, Spain, July 2007 (unpublished). 